\title{
Comparative analysis of assessment of the craniocervical equilibrium through two methods: cephalomotry of Rocabado and cervical range of Motion
}

\author{
Lilian Becerra de Oliveira, PT, AMPT ${ }^{\mathrm{a}}$; Franklin Cajaíba, $\mathrm{PT}^{\mathrm{a}}$; Wesley Costa, $\mathrm{PT}^{\mathrm{a}}$; Mariano \\ Rocabado, PHD ${ }^{\mathrm{b}}$; Rodrigo Lazo-Osório, $\mathrm{PHD}^{\mathrm{c}}$; Sanzia Ribeiro, PT, MS ${ }^{\mathrm{a}}$ \\ ${ }^{a}$ Faculdade Adventista da Bahia, Caixa Postal No 18 CEP 44.300-000 Cachoeira, Ba. BRAZIL. \\ ${ }^{\mathrm{b}}$ University Andres Bello, Santiago, Chile. ${ }^{\mathrm{C}}$ University Vale do Paraiba, Brazil. olilian@hotmail.com
}

\begin{abstract}
There are several instruments of evaluation of the craniocervical equilibrium; the most reliable are the radiographies. This study used the cephalometric analysis of Rocabado to measure the sensibility and specificity of the Cervical Range of Motion (CROM), a goniometer designed to assess cervical movements in degrees, and measure the forward head position in centimeters. This instrument frequently used, has been tested as a reliable instrument to evaluate the cervical movements but not the forward head. The sample consisted of 30 volunteers, 18 females, 12 males, mean age of 24.63 years. All participants were evaluated with CROM and radiographies in the resting head position and in erect head position. The values considered by the cephalometry consisted in the angle made between the McGregor plane and the vertical line formed by the base of the odontoid process to its apex; the posterior space between $\mathrm{C} 0-\mathrm{C} 1$ and $\mathrm{C} 1-\mathrm{C} 2$ and the hyoid triangle. Results: $30 \%$ of the subjects had forward head posture, according to de cephalometry of Rocabado (decreased space between $\mathrm{C} 0-\mathrm{C} 1, \mathrm{C} 1-\mathrm{C} 2$ ) and $43,3 \%$ according to CROM. 16,6\% had decreased posterior-inferior angle, and $13 \%$ had the hyoid triangle facing up. ROC curve of identifying forward head posture yielded area under the curve of 0,778 (95\% confidence interval $0,596-0,960)$. The sensibility of CROM was: $77 \%$. The specificity $71 \%$. Conclusion: This study suggests that CROM has a moderate sensibility and specificity, useful for clinic use, but not for research.
\end{abstract}

Key words: cephalometry, CROM, forward head posture

\section{Introduction}

The American Orthopedics Academy describes the posture as a balance between muscles and bones that protects the structures of the body from traumas in every position. [1]

The correct posture is the position where minimum stress is applied on the joints, keeping it from fatigue. The posture is a dynamic situation. The body parts adapt itself to the great variety of stimulation received, reflecting the momentary experiences. [2][3]

The posture of each individual is determined by the muscle chains, fascia, ligaments and bone structure that have a coordinated system and are interdependent among each other and reach the entire organism. [4]
The interdependence of the components of the human body makes it that the minor anomaly of the supporting structures induces a postural disharmony. This way, an initial tension in the muscle chains is responsible for a succession of tensions on the associated tendons. When a muscle shortening happens, extremities approach shifting the bones in which they are inserted into, thus, the joint blocks and the body deforms. [5]

Maintenance of the correct posture demands an adequate muscle tone and flexibility because the muscles need to work constantly against the gravity and in harmony with the other muscles. So, incorrect posture becomes responsible for compensation between different muscular groups compromising several of their functions. [3] 
Many assessment methods have been used to measure the stability of the craneocervical articulation, to quantify the musculoskeletal deficit, and also to serve as a base for the assessment of the efficiency of the therapeutic interventions. Among the most known are: goniometer, inclinometers, visual estimation, tape measurements, photographic method and teleradiography (X-ray) with its cephalometry. [6] [7]

One of the first cephalometric analyses in teleradiography was described by Rocabado. [8] Other studies have used this method, like Zepa [9] who studied the association between the thoracic kyphosis, posture of the head and craniofacial morphology in young adults. Figun [10] compares the results considered normal (in Caucasian subjects) with Mapuche subjects. Abreu [11] analyzed the results of the cephalometric in children and adults with temporomandibular dysfunction. It is understood that the assessments by the X-ray are the most trustable to analyze the craneocervical balance, being the base diagnosis for this research.

The Cervical Range of Motion Goniometer $(\mathrm{CROM})$ is an instrument of measurement in degrees that assess all movements of the head and the forward head posture. Its creation was motivated by the necessity of an instrument easy to use in the clinic for the objective record of the patient evolution. The measurements were validated in degrees of flexion, extension, right and left rotation and lateralization of the cervical spine by Tousignant [12] Recommended by Chantal [6] after a systematic review. This is so, even if the literature doesn't present studies that validate the measurement of the forward head posture.

This study pretended to analyze the sensibility and the specificity of the CROM when compared to the X-ray in the assessment of the forward head posture with the objective to validate its utilization in clinical practice.

\section{Method}

This is a study of accuracy, analytic, of transversal character. This work was done at the Faculdade Adventista da Bahia, Cachoeira-Ba, having as subjects the students of the physical therapy course. The X-rays were done at the Cleriston Andrade General Hospital (CAGH), situated in the city of Feira de Santana-BA.

\subsection{Participants.}

Thirty asymptomatic subjects participated in this study, students of the Physical Therapy course, at the Faculdade Adventista da Bahia, being 18 females and 12 males with ages ranging from 18 to 35 years old (average age 24,6 years old). All subjects signed an informed consent, approved by the ethics committee and research of the Faculdade Adventista da Bahia. Subjects with a diagnosis of temporomandibular dysfunction (TMD) or neck pain, subjects with any kind of osteomioarticular pain or that had realized any physical therapeutic and/or orthopedic treatment in the last three months were excluded.

\subsection{Procedures.}

The data collection was done at the Cleriston Andrade General Hospital in the period of July to September, 2009. The instrument used for goniometric measurement was CROM, introduced on the market by Performance Attainment Associates. It measures flexion, extension, lateral flexion, cervical rotation and forward head posture using two inclinometers with gravity-dependent needle in the sagital and frontal planes. In the horizontal plane the needle is magnetic. An extra bar having in its extreme an inclinometer measures de forward head posture in centimeters.

The measurement of the forward head posture is obtained from the intersection of a bar positioned vertically over the spinal process of the $7^{\text {th }}$ cervical vertebra (C7), with another horizontal bar connected to the instrument. The value of the forward head posture is obtained by the subtraction of the values collected in the head resting position and the erect head position, assisted by the therapist. [12] (Figure 1).

For the X-rays of the cervical in profile, the X-ray (Yoshida Kaylor) was used, radiographic film IBF Medix TMG/RA, with the dimensions of 24 x $30 \mathrm{~cm}$. automatic processor model (Air Techniques) and lead apron.

Two X-rays of the cervical in profile of each subject was done by the same operator, one in the rest position and another in the erect head position, as the protocol created by the evaluators indicated. All volunteers were guided to assume the rest and erect head positions by the evaluator.

The protocol used to obtain the X-rays was following the protocol adopted by Sakai [13] and it was the following: the patient was sitting in a white plastic chair, straight back, with feet slightly apart (approximately $20 \mathrm{~cm}$ ), with the palm of the hands on the anterior face of the thigh. CROM was placed on the voluntary and it was requested that the subject would stay in the rest position. The position was verified by only one evaluator who measured the quantity of degrees obtained through the CROM without any correction of compensations done by the volunteer. Following that, the X-ray was taken. 
Next, a verbal command requested the volunteer to put his head in the erect head position (the researcher positioned the head in case he/she didn't understand the instruction) and another

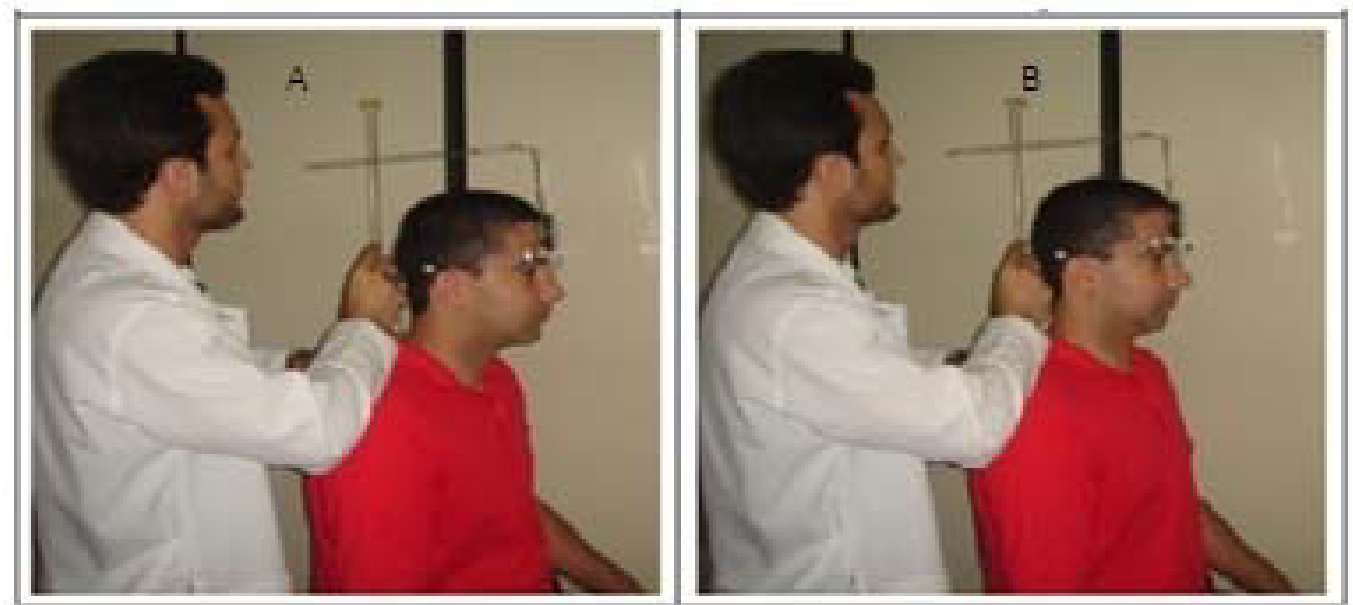

Figure 1: CROM A: Resting Head Position. B: Erect Head Position
For obtaining the cephalometry the craneocervical anatomic structures were drawn as recommended by Rocabado[8] (Figure 2), they are: a posteroinferior angle, formed in the posteroinferior quadrant of the tangential lines of the McGregor plan and the line that unites the margins anterior part of the interior part of the body of the axis to the apex of the odontoid process (normal value $101^{\circ} \pm 5^{\circ}$ ). The space between $\mathrm{C} 0-\mathrm{C} 1$ (occipital line and the first cervical vertebra) and C1-C2 (space between the first and second cervical vertebra), (normal values $6,5 \mathrm{~mm} \pm 4-9 \mathrm{~mm}$ ). (Figure 3 ) The hyoid triangle formed by the lines created between the distance from the most posterior and inferior of the jaw to the closest to the hyoid bone, the line from the most posterior and inferior of the jaw to the closest to the hyoid bone, the line formed between the hyoid bone and the inferior and anterior margin of $\mathrm{C} 3$ and the line between $\mathrm{C} 3$ and the jaw.

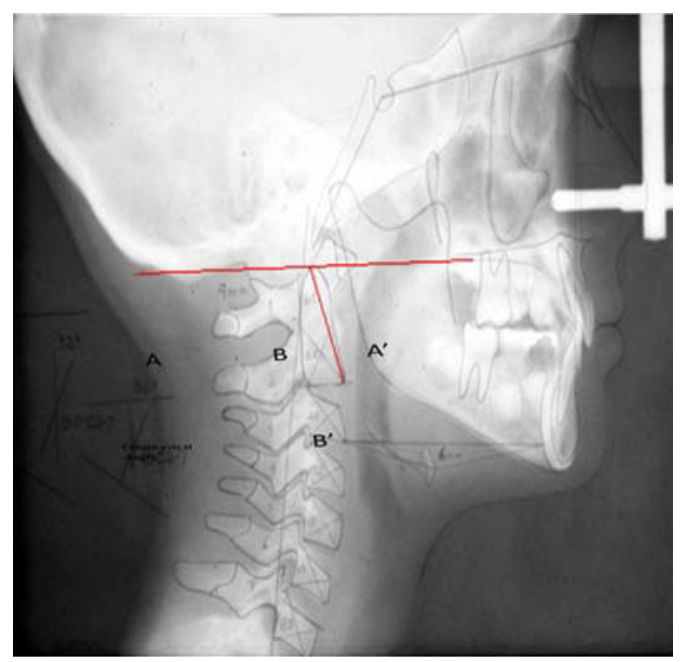

data collection was made using CROM. Then another X-ray was taken with the head erect position. cephalometry of Rocabado formed by the crossing of the MacGregor plane A-A and the Odontoid plan B-B.

For better visualization of anatomic structures, the tracing were made in a completely dark room using a table light box. The tracing together with the measure of the angulation of the spinal spaces were realized only by one evaluator (Figure 3).

Other material used for the research: 01 laptop (Sony Vaio); one multifunctional printer (HP Deskjet F380); one digital camera (SONY Cyber-shot, model DSC-T5); one Ruler (FABER CASTELL black with $20 \mathrm{~cm}$ ); one Medium paint brush (red and black); one Small scotch tape.

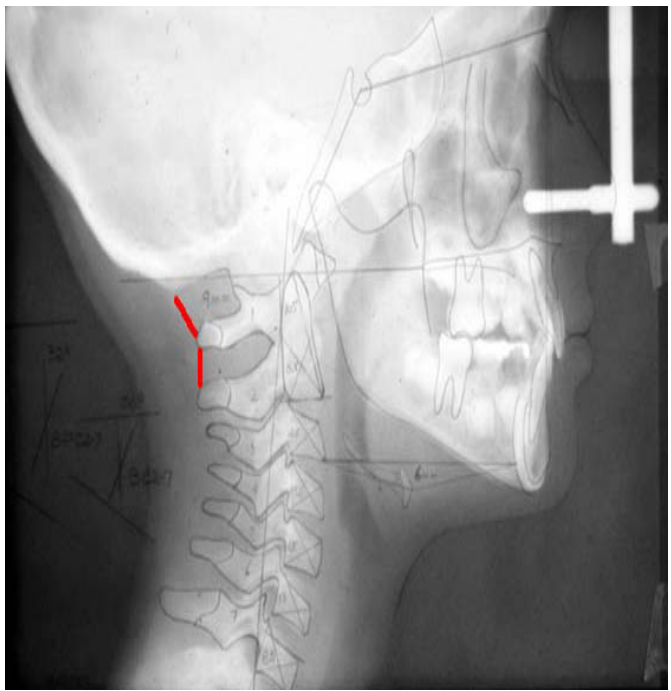

Figure 3. Distance 0 -A equal to $\mathrm{C} 0-\mathrm{C} 1$ according with the cephalometry of Rocabado where 0 is the base of the occipital and A, the most superior point of the arc of the atlas. 


\subsection{Statistical analysis}

The primary outcome studied in this work were: number of subjects with forward head posture by the decrease of the spaces $\mathrm{C} 0-\mathrm{C} 1, \mathrm{C} 1$ $\mathrm{C} 2$ of the Rocabado's tracing[8], compared to analytic paired test, of the number of subjects with forward head posture by CROM, in order to analyze sensibility and specificity and use of the ROC curve.

Secondary outcomes: number of subjects that presented the angle posterior-inferior decreased and the position of hyoid triangle, according to the Rocabado's tracing [8]; comparison of the means of the values obtained by the posteriorinferior angle in the resting position and the erect head position, according to the cephalometry of Rocabado; comparison of the mean of the addition of the values of the spaces between $\mathrm{C} 0$ $\mathrm{C} 1$ and $\mathrm{C} 1-\mathrm{C} 2$ in the resting and erect head position.

The sample was calculated by the statistic program WinPepi, with a significant level of $0,5 \%$, a power of $80 \%$ and a SD of 8,1 .

\subsection{Results}

The group with forward head posture $(n=9)$ consisted of 5 women and 4 men with a mean age of 26,2 years old. Without forward head posture 21 subjects, 8 men and 13 women with mean age of 23 years old. (Table 1). With a significant difference between groups, ChiSquare test, $p=0,028$. The forward head position group was identified by the cephalometry of Rocabado when evaluated the spaces between $\mathrm{C} 0-\mathrm{C} 1, \mathrm{C} 1-\mathrm{C} 2$. It is understood that there is not a decrease of cephalometry of spaces (that occurs with a posterior rotation of the skull) without forward head posture, by the natural tendency of maintaining the vision in the horizontal plane.

Table 1

Demographic characteristics of subjects with and without forward head posture (defined by cephalometric analysis of

\begin{tabular}{l|c|c}
\hline \multicolumn{3}{c}{ Rocabado (n=30) } \\
\hline Variable & $\begin{array}{c}\text { Diminish } \\
\text { space } \\
\text { between C0- } \\
\text { C1-C1-C2 }\end{array}$ & $\begin{array}{l}\text { Normal } \\
\text { space } \\
\text { between C0- } \\
\text { C1-C1-C2 }\end{array}$ \\
\hline Age & 26,2 & 24 \\
Female & $5(55 \%)$ & $13(62 \%)$ \\
Male & $4(44 \%)$ & $8(38 \%)$ \\
Caucasian & $9(100 \%)$ & $21(100 \%)$ \\
CROM (Mean) & 3,33 & 2,0 \\
\hline
\end{tabular}

The values obtained for Sensibility were $77 \%$ and Specificity 71\%. Positive Predictive Value $\mathrm{PPV}=53 \%$, and the Negative Predictive Value $\mathrm{NPV}=88 \%$. The result of the Accuracy was
$\mathrm{A}=73 \%$. With a reason of Positive Likelihood $=$ 2,65. Reason of negative Likelihood $=0,23$. (Table 2). It was analyzed with a paired $\mathrm{T}$ test, the values obtained in the posteroinferior angle in the rest position and forward head position for the measurement analysis.

Table 2

Sensibility, specificity, PPV, NPV and accuracy.

\begin{tabular}{|l|c|c|c|}
\hline \multirow{2}{*}{} & \multicolumn{2}{|c|}{$\mathrm{C} 0-\mathrm{C} 1, \mathrm{C} 1-\mathrm{C} 2$} & \multirow{2}{*}{ total } \\
\cline { 2 - 4 } & With & Without & 13 \\
\hline $\begin{array}{l}\text { CROM with forward head } \\
\text { posture }\end{array}$ & 7 & 6 & 17 \\
\hline $\begin{array}{l}\text { CROM without forward } \\
\text { head posture }\end{array}$ & 2 & 15 & 30 \\
\hline Total & 9 & 21 & \\
\hline
\end{tabular}

Forward head posture was identified in 13 subjects from $30(43,3 \%)$ through CROM; also five subjects $(16,6 \%)$ presented craniocervical unbalance with a decrease angle posteroinferior formed by the posteroinferior quadrant of the intersectional lines of the McGregor plane and the line of the odontoid plane inferior to $101^{\circ}$.

The ROC curve constructed from the graphic representation of the values of sensitivity and specificity founded, is presented in the Figure 4. The cut point was found at 0.778 (95\% confidence interval 0,596-0,960).

ROC Curve

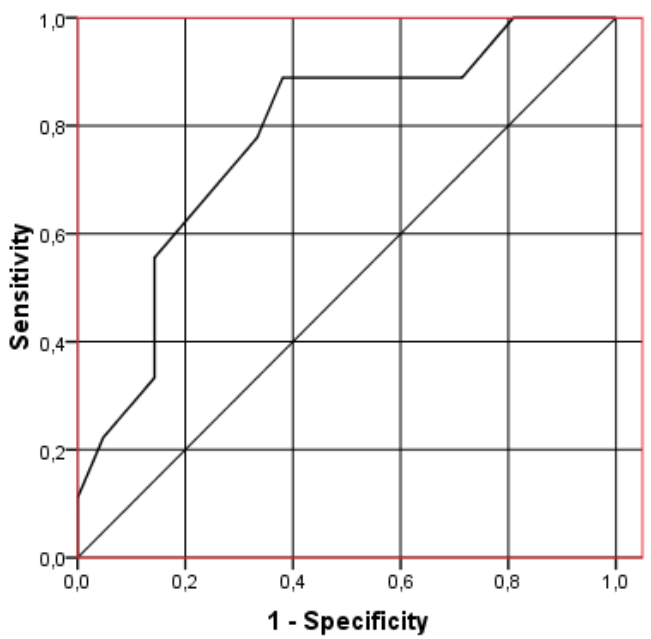

Figure 4. ROC curve. Forward head posture identified by CROM according to the Rocabado Cefalometry.

With paired $\mathrm{T}$ test, the values obtained from the posteriorinferior angle in the rest and erect head position were analyzed. The means of the resting head position were: 105,7 (Std. Deviation $=7,4)$ and the mean for the erect head posture was $108,7($ Std. Deviation $=8,1)$ with a $p=0,00$. The means of the values obtained from the spaces of $\mathrm{C} 0-\mathrm{C} 1$ and $\mathrm{C} 1-\mathrm{C} 2$ in the resting and erect head posture, were compared. The mean value of the rest position was $1,6 \mathrm{~cm}$. and the mean value of the spaces in erect head posture 
was of 1,9 (Std. Deviation=0,27). with a $\mathrm{p}=0,000$.

\subsection{Discussion}

The CROM demonstrated a moderate sensibility and specificity in the evaluation of the forward head posture of the head when compared to the decrease of the spaces $\mathrm{C} 0-\mathrm{C} 1, \mathrm{C} 1-\mathrm{C} 2$ of the Cephalometry of Rocabado. There aren't any references for these datas to be compared and discussed, even that there are several studies about CROM evaluating the inter and intratesters reliability, and the validation of the instrument, for the measurements of head flexion and extension, cervical lateralization and right and left rotations. [13][14][15][16] $43 \%$ of subjects were identified by the CROM as having forward head posture, Griegel-Morris[17] identified in an asymptomatic adult with the ages between 20-50 years old a prevalence of forward head posture of $66 \%$, the difference in the value can be in fact that the age average in both groups were different 36 years old in comparison with the average in this study of 24,6 years old. $16,6 \%$ of the subjects had a reduction in the posteroinferior angle, even that it was expected a higher percentage. $20 \%$ presented normal angulation $\left(101^{\circ} \pm 5^{\circ}\right)$ and $63,3 \%$ presented a rectification of the cervical spine, that may lead to degenerative process in the medium and low cervical spine. [18] Four subjects evaluated presented an inverted hyoid triangle, not having any relation, if this happens more frequently in subjects with an anterior or posterior rotation of the cranium. These results may be due to the size of the sample, being a random outcome or it may really have a direct relation due to the subjects having different tensions and measurements of the hyoid muscles as evidenced by Iunes [19] and Matheus[20]. It was expected that the average of the degrees of the posterior part of the inferior angle would be greater when compared to subjects in the rest and erect head position, by a bigger flexibility of the vertebral segments awaited by the average ages, however, with such similar results it motivates new hypothesis. The high number of subjects with rectification of the cervical spine also leads to other studies to investigate this findings with a bigger number of subjects.

Limitations: this study did not evaluate the level of corporal mass that could affect the measurements of CROM.

\section{Conclusion}

The sensibility of the CROM for the evaluation of the forward head posture, showed to be moderate $\mathrm{S}=$ was analyze $77 \%$ with a lesser specificity $71 \%$, useful for the daily data of clinical practice, but, for research, more reliable values are found with the use of the X-ray.

\section{References}

[1] L.Braccialli and R. Vilarta, Postura corporal: reflexões teóricas, Fisioterapia e Movimento 14 (2001), 65-71.

[2] D. Magee, Avaliação musculoesquelética, Manole, Barueri, 2006, pp. 837.

[3] A.Cesar, et. al, A influência da postura anteriorizada na cabeça no padrão mastigatório, CEFAC 6 (2004), 259265.

[4] P.F. Kendall; E.K Mccreary ;P.G Provance, Músculos, Provas e Funções, Manole, São Paulo, 2007, pp 172.

[5] D. Amantéa. et. al, A importância da avaliação postural no paciente com disfunção da articulação temporomandibular, ACTA Ortopédica Brasileira12 (2004), 155-159.

[6] H.P. Chantal, Clinimetric evaluation of active range of motion measures in patients with non-specific neck pain: a systematic review, Europen Spine Journal (2008). 905-921.

[7] W. Rheault; B. Albright; C. Byers et al. Intertester Reliability of the cervical range of Motion Device, JOSPT 15(1992), 147-150.

[8] M.S. Rocabado. Relaciones biomecânicas de las regiones craneales, cervicales e hióideas. Ortodoncia, 58 (1994) 51-56.

[9] I. Zepa; I.K. Hurmerinta; O.Kovero; N. Maunu; M. Kononen, Associations between thoracic kyphosis, head posture, and craniofacial morphology in young adults, ActaOdontologic Scandinavian Journal 58 (2000), 237-242.

[10] M.E. Fígun; R.R. Garino, Artrologia. In: Anatomía Odontológica Funcional e Aplicada. Médica Panamericana, São Paulo, 1994, pp.44-60.

[11] G. Abreu; A. Albino; M. Bim; M Rocabado; R. Cecílio et al. Análise cefalometrica em crianças e adultos com disfunções temporomandibulares. IV Encontro Latino Americano de Iniciação científica, UNIVAP, (2007), 1519-1521.

[12] M. Tousignant et. al. Criterion validy of the cervical range of motion (CROM) goniometer for cevical flexion and extension, Spine 25 (2000), 324-330.

[13] E. Sakai, Avaliação eletromiográfica de músculos da mastigação em pacientes portadores de maloclusão tratados com ortopedia funcional dos maxilares, Ph.D. Dissertation,Faculdade de Odontologia de Piracicaba, 2006.

[14] D. Capuano-Pucci; W. Rheault; J. Aukai; M. Bracke et al. Intratester and intertester reliability of the cervical range of motion device, Arch Phys Med Rehabil72 (1991), 338-340.

[15] W. Rheault; B. Albright; C.Beyers et al Intertester reliability of the cervical range of motion device, $\mathrm{J}$ Orthop Sports PhysTher 15 (1992) 147-150.

[16] N.R. Ordway; R. Seymour; R.G. Donelson, et al, Cervical sagital range-of-motion analysis using three methods.Cervical range-of-motion device, 3space, and radiography, Spine 22 (1997) 501-508.

[17] P. Griegel-Morris; K. Larson; K. Mueller-Klaus and C. A. Oatis, Incidence of common postural abnormality in the cervical shoulder; and thoracic regions and their association with pain in two age groups of healthy subjects, Physical Therapy, 72 (1992) 425-431.

[18] M. Rocabado. Distúrbios da ATM. São Paulo, 2008. 12 f. Notas de aula.

[19] D.H. Iunes; L. Carvalho; A.S. Oliveira and D. Bevilaqua-Grossi, Craniocervical posture analysis in 
patients with temporomandibular disorder, Rev. Bras

Fisioter. 13 (2009) 89-95.

[20] R. A. Matheus; F. M. Ramos-Perez; A. V. Menezes et al,

The relationship between temporomandibular dysfunction and head and cervical posture, J Appl Oral Sci, 17 (2009) 204-208. 